\title{
Modeling damage accumulation to fracture in a magnesium-rare earth alloy
}

\author{
B. Kondori ${ }^{1}$ and A. A. Benzerga ${ }^{1,2}$ \\ ${ }^{1}$ Department of Materials Science \& Engineering, Texas A\&M University, College Station, TX 77843, USA \\ ${ }^{2}$ Department of Aerospace Engineering, Texas A $3 M$ University, College Station, TX 77843, USA
}

October 15, 2016

\begin{abstract}
A micromechanics-based continuum damage model is employed to rationalize key experimental trends in the fracture of a Mg-Y-RE alloy. The model has three components: (i) a phenomenological law of strain-controlled void nucleation; (ii) a void growth part that accounts for microcrack blunting; and (iii) a void coalescence part that determines fracture. Plastic flow in the undamaged state is represented by $J_{2}$ theory with isotropic hardening. The material parameters involved in the model consist of (i) the hardening parameters, (ii) the initial values of microstructural variables associated with damage, and (iii) one adjustable parameter entering the microcrack opening rate equation. An experimental procedure for determining the material parameters is outlined. The model enables several hypotheses to be explored. It is found that when the volume fraction, aspect ratio and spatial arrangement of initially nucleated microcracks are associated with second-phase particles on the grain boundaries, the fracture strains are well predicted over a range of stress triaxialities using a single heuristic parameter that governs the rate of microcrack blunting. Implications are discussed in the broader context of high-strength materials failing after limited void growth.
\end{abstract}

Keywords: Ductility; Stress triaxiality; Micromechanics; Model identification; Fracture strain

\section{Introduction}

The insertion of magnesium alloys as structural materials hinges upon the improvement of their low temperature formability and ductility. Limits to these are believed to stem from pronounced micro- and macroscale anisotropies, which result from the crystal structure and processing texture, respectively [1,2]. Accordingly, $\mathrm{Mg}$ alloys with suppressed plastic anisotropy would be more ductile than those having a sharp processing texture, such as 
AZ31 and ZK60. The validity of such hypothesis, however, remains to be investigated. Owing to their weakened texture and reduced plastic anisotropy, magnesium alloys with rare-earth (RE) additions are believed to be promising alternatives to commercially available $\mathrm{Mg}$ alloys [3-6]. With sufficiently high concentrations, RE containing $\mathrm{Mg}$ alloys, such as WE43, are stronger than commercial ones with no loss in ductility either in simple tension or compression [7]. Combination of isotropic behavior, high strength and good tensile ductility makes these alloys attractive candidates for automotive and aerospace industries as well as in armour applications.

To accelerate the insertion of Mg-RE alloys as structural materials, investigating their ductile fracture behavior under various stress states, not merely restricted to uniaxial loading conditions, is much needed. The fracture behavior of metals is known to be significantly affected by the stress state triaxiality, which may be quantified by the ratio of mean normal stress to effective stress [8]. However, investigating such effects has not been carried out systematically for $\mathrm{Mg}$ alloys. In a study dedicated to triaxiality effects, it was shown that the notch ductility of AZ31B with strong basal texture is higher than its tensile ductility $[9,10]$. The maximum strain-to-failure in AZ31B was obtained at moderate triaxiality. After this maximum, the ductility was found to decrease with further increase in triaxiality, a trend that is consistent with void growth and coalescence mechanisms. Three factors were identified to influence the obtained fracture locus. First, the topography of fracture surfaces indicated a change from twin-induced cracking under uniaxial loading to void nucleation on second phase particles in notched bars. Second, a transition form shear-like fracture in smooth bars to normal fracture in notched ones was noted. Finally, activation of additional deformation mechanisms, such as extension twinning and $\langle c+a\rangle$ slip at high triaxialities, were rationalized in [9] and further explored using crystal plasticity simulations in [11].

Recent experiments also showed a significant sensitivity of ductility to the stress state in $\mathrm{Mg}-\mathrm{RE}$ alloys [7,12]. In particular, the authors have shown in [12] that the notch sensitivity of WE43 alloy is much stronger than that of AZ31B. Post-mortem fractography revealed instances of grain pull-out at all stress triaxialities with the density of grain pull-out increasing with triaxiality. This indicated a mode of ductile intergranular fracture (IGF). It was further hypothesized in [12] that the second phase particles and precipitates that decorate grain boundaries played a key role in promoting IGF. Davidson et al. [13] studied the effect of hydrostatic pressure on fracture of hot-extruded pure magnesium. They reported that ductility initially increases linearly with pressure, a behavior attributed to prevention of either IGF or void nucleation and growth. A reverse effect can be envisioned in the notched bar experiments of Ref. [12] where elevated hydrostatic tension at higher triaxialities facilitates IGF and void coalescence. It remains to be seen whether the fracture locus in stress space can be rationalized on the basis of an IGF induced by the grain-boundary particles. The aim of this paper is to develop a model as a first step in this direction.

To date, a few experimental research efforts have been dedicated to the identification of damage initiation mechanisms, such as deformation twins [14-16] or second phase particles $[9,10,17]$. Other experimental studies have investigated the effect of a macroscopic main crack on the deformation systems in their immediate vicinity [18-20]. Most of the research motivated by understanding damage and fracture in $\mathrm{Mg}$ alloys has focused on their complex deformation behavior, viewing fracture but as an end to the deformation process or a mere consequence of attaining a critical strain state. In this regard, the fracture mechanics 
community has developed sophisticated models for ductile failure in the past several decades; see recent reviews $[8,21,22]$. Some of these models are able to accurately predict the interaction between voids and microcracks in an anisotropic medium and render valuable insight on microstructural evolutions after damage initiation. For instance, Benzerga et al. [23] implemented constitutive relations for coupled plasticity and anisotropic damage and carried out three-dimensional simulations of crack initiation and growth in notched bars. However, application of such modeling approaches to $\mathrm{Mg}$ alloys is still lacking.

In this paper, detailed fractography is performed to investigate the extent to which grainboundary particles are involved in IGF. A micromechanics-based analysis of void nucleation, growth and coalescence is used to relate initial microstructural variables in WE43 and their evolution to the observed fracture behavior. The analysis follows a methodology similar to that used by Pardoen et al. [24] to rationalize intergranular versus transgranular fracture in aluminum alloys. In addition, the analysis employs a fully analytical coalescence criterion and invokes only one fitting parameter that modifies the evolution of void shape. Using this modeling strategy, the model predictions for stressing paths that mimic actual ones in notched specimens are assessed against experimental measurements made in [12].

\section{Experimental procedure}

\subsection{Material and Microstructural Analyses}

The starting material is a hot-rolled plate of WE43 in T5 condition (strain hardened and artificially aged) with thickness of $1.5 "(38 \mathrm{~mm})$. The nominal composition of the plate is given in Table 1. The principal directions of the plate are labeled L (longitudinal/rolling), $\mathrm{T}$ (transverse) and S (short-transverse/normal). Metallographic sections were mechanically ground using $\mathrm{SiC}$ paper and fine polished using 1, 0.3 and $0.05 \mu \mathrm{m}$ alumina suspensions. The use of water was restricted to grinding only. For rinsing and ultrasonic cleansing, Isopropyl alcohol and acetone were respectively used. An acetic picral solution (4.2 g picric acid, $10 \mathrm{ml}$ acetic acid, $70 \mathrm{ml}$ ethanol and $10 \mathrm{ml}$ water) was used as etchant for about $5 \mathrm{~s}$. Optical, Scanning Electron Microscopy (SEM) and Energy Dispersive X-Ray Spectroscopy (EDS) were used for microstructural characterization. Grain sizes were measured using the line intercept method [25] and corroborated by area measurements using the image analysis software "Image J". Crystallographic texture measurements were carried out using a BrukerAXS D8 X-ray diffractometer (XRD) with $\mathrm{Cu} \mathrm{K} \alpha$ radiation on a sample from the plate's mid-section to get (0002) and (1010) pole figures using a $5^{\circ}$ grid size and an $85^{\circ}$ sample tilt.

\subsection{Mechanical Behavior and Anisotropy}

Cylindrical smooth and notched tensile bars were used to study the effect of stress state on fracture. Various notch acuities were introduced to provide a range of stress triaxialities $[12,26]$. Cylindrical specimens were exclusively used to reduce the propensity to shear localization. Bars were initially cut along the rolling direction (L) using an Electric Discharge Machining (EDM) wire then machined to final shape. The $\mathrm{S}$ direction was systematically marked on both ends of each specimen to enable the measurement of deformation anisotropy. 
The specimens were deformed to complete fracture. Uniaxial tension experiments were carried out at an initial strain rate of $10^{-3} \mathrm{~s}^{-1}$ on a servo-hydraulic MTS machine (Model 380.50 ) equipped with a $250 \mathrm{kN}$ load cell. True axial strain was measured using a laser extensometer over a gauge length of $30 \mathrm{~mm}$. The tests were interrupted at different strain intervals to measure the lateral strains along two perpendicular directions (at least one principal direction) using a caliper while the sample was held under fixed grip condition. Lateral strains are calculated as

$$
\varepsilon_{\mathrm{X}}=\ln \left(\frac{\Phi_{X}}{\Phi_{0}}\right)
$$

where the superscript 0 refers to initial diameters. The accuracy on strains $\left(\varepsilon_{\mathrm{X}}\right)$ is $0.1 \%$. The area of the fractured specimen, $A_{\mathrm{f}}$, was measured post-mortem using top-view photographs of the fractured specimen assuming an elliptical shape. As a measure of ductility, the strain to complete fracture was defines on the basis of cross-sectional area variation:

$$
\varepsilon_{\mathrm{f}}=\ln \left(\frac{A_{f}}{A_{0}}\right)
$$

Round notched $(\mathrm{RN})$ can be characterized based on the notch severity parameter, $\zeta$, equal to ten times the notch radius to specimen diameter at the notch. Three values of $\zeta$ were explored and the corresponding specimens were denoted by $\mathrm{RN} \zeta$. There is a direct relation between notch severity and stress triaxiality. The lower the value of $\zeta$ the higher the levels of stress triaxiality. Taking the notch height as a gauge length, a nominal strain rate of $3 \times 10^{-4} s^{-1}$ was imposed in all cases. The instantaneous diameter along $\mathrm{S}$ direction was continuously measured thanks to a custom-made radial extensometer [9].

Unlike in initially smooth tensile bars, the plastic strains are spatially nonuniform in the gauge section of a notched bar. Hence, the following definitions are typically adopted; see Ref. [26]:

$$
\begin{gathered}
\bar{\varepsilon}_{\mathrm{X}}=\ln \left(\frac{\Phi_{\mathrm{X}}}{\Phi_{0}}\right), \\
\bar{\varepsilon}_{\mathrm{f}}=\left.\bar{\varepsilon}_{\mathrm{S}}\right|_{\mathrm{f}}+\left.\bar{\varepsilon}_{\mathrm{T}}\right|_{\mathrm{f}},
\end{gathered}
$$

where the bar stands for spatial averaging over the minimum-diameter section (absolute values taken where appropriate). Two specimens for each triaxiality are tested and results show small scatter.

\section{$2.3 \quad$ Fractography}

The fracture surfaces of broken specimens were sprayed with a silicone mold release spray immediately after each fracture test in order to prevent oxidation. The specimens were then held in a manually vacuumed desiccator prior to being examined in SEM. It is worth noting that, even with extreme care, the propensity for oxidation is such that fracture surfaces can only be observed once. For this reason, the testing campaign has been paced to accommodate SEM observations of oxide-free fracture surfaces. Occasionally, EDS analysis of the second phases on the surface was recorded. 


\section{Experimental results}

Figure 1a depicts a 3D rendering in optical microscopy of the initial microstructure of WE43. The microstructure exhibits a majority of equiaxed grains with some pancake-like grains occasionally observed in planes containing the through-thickness direction. The grain size measurements carried out in [12] over a population of $\sim 350$ grains suggests a mild dual grain size distribution with $\approx 10 \mu \mathrm{m}$ small grains and $\approx 25 \mu \mathrm{m}$ larger grains, on average. Macroscopic texture measurements show that alloying elements of WE43 significantly alter its texture (Fig. 1b) in comparison with pure magnesium or alloys such as AZ31 or ZK60. The present alloy exhibits a weak texture and the basal pole is also shifted towards the rolling (L) direction, consistent with the literature [27-29].

A 3D representation of the microstructure in SEM is depicted in Figure 2, at a somewhat finer scale, so as to reveal the distribution of second phase particles. The majority of particles are located near or at grain boundaries whereas few particles are found in the grain interiors. The high number density of grain-boundary particles has been reported in [30] and is rationalized by the segregation of alloying elements at these interfaces [5]. Particle clusters are also noted, e.g., in T-S planes and are aligned perpendicular to the through-thickness (S) direction. Nano-sized precipitates have also been reported in the litarture [30,31] but are not visible in the SEM micrographs. Using EDS analyses (not shown for brevity) and information from the literature [32-35], the second phase particles in the present alloy are qualitatively identify as $\mathrm{Mg}_{41} \mathrm{Nd}_{5}, \mathrm{Mg}_{2} \mathrm{Y}, \mathrm{Mg}_{24} \mathrm{Y}_{5}, \beta-\mathrm{Mg}_{14} \mathrm{Nd}_{2} \mathrm{Y}$ and $\mathrm{Mg}-\mathrm{Zr}$. The white contrast bands, perpendicular to the $\mathrm{S}$ direction, are also identified as Zr-rich regions; see [12] for details.

The true stress-strain tensile response is shown in Figure 3a and compared with the response in compression from [12]. The tension-compression asymmetry, which is pronounced in pure $\mathrm{Mg}$ and alloys such as AZ31, is not observed in WE43. Also, the compression responses along the three principal directions published in [12] showed negligible anisotropy in flow strength. It was also reported in [12] that in tension the lateral strains, as defined by equation 1, were independent of direction so that initially circular cross sections remained circular during deformation. Here, this is quantified further in Figure 3b, which shows the evolution of the anisotropy ratio $\left(R=\varepsilon_{T} / \varepsilon_{S}\right)$. Data for AZ31B alloy from [9] is also shown for comparison. This result corroborates the qualitative observations in [12]. It also shows that before a quasi-isotropic steady-state is reached there is a transient (up to a strain of $\sim 0.05)$ where larger diameter reductions are observed along the through-thickness (S) direction compared to transverse $(\mathrm{T})$ direction. According to the texture, presented in Figure 1b, a moderate majority of the basal plane normals are oriented at $45^{\circ}$ between $\mathrm{L}$ and S. Ease of glide for $\langle a\rangle$ dislocations on these basal planes rationalizes $R^{L}<1$ at the early stages of deformation. From the results of this figure along with those in Ref. [12], it is concluded that mechanical response of wrought WE43 in tension is isotropic.

For the sake of comparison with modeling predictions below, key results on the notched bar tests carried out in [12] are summarized in Figure 4. Inside the notch the stress state is triaxial which means that in addition to the major axial stress, $\Sigma$, there are two equal minor (principal) stresses, each denoted by $\sigma<\Sigma$. The sharper the notch the higher the ratio $\sigma / \Sigma$. The effect of stress triaxiality on the load versus diameter reduction response is shown in Figure 4a whereas the locus of strain to failure versus nominal triaxiality, inferred 
from $\mathrm{FE}$ analysis of an isotropic materials, is shown in Figure 4b. Note that, according to equation 4, the strain to failure includes the contribution of both lateral strains, not only the one measured continuously and reported in part (a). For WE43 alloy, since deformation anisotropy is minimal the total strain to failure is roughly twice that associated with a single lateral strain, as per equation 3. However, for AZ31B alloy, the results of which are also included in Figure 4b for comparison, the two contributions are largely different, see [9] for details. In both the smooth and notched tensile bars, fracture occurred abruptly with no visible load drop.

The result presented in Figure 4 shows that strain to failure in WE43 depends strongly on the stress-state triaxiality. The average value of area reduction in WE43 specimens is reduced from $\bar{\varepsilon}_{f}=0.156$ in RN10 specimens to $\bar{\varepsilon}_{f}=0.042$ in RN2 notched bars. As in AZ31, the fracture locus of WE43 does not exhibit its maximum under uniaxial tension. The fracture strain of WE43 under uniaxial loading is $\bar{\varepsilon}_{f}=0.126$. According to this figure, WE43 and AZ31 exhibit significantly different sensitivity to triaxiality. Despite comparable ductility under uniaxial loading, WE43 offers much lower ductility than AZ31 under triaxial loading (e.g., in RN2 specimens $\bar{\varepsilon}_{f}^{A Z 31} \approx 4 \times \bar{\varepsilon}_{f}^{W E 43}$ ). Note that in this comparison AZ31 is an alloy with pronounced anisotropy, tension/compression asymmetry and strong basal texture whereas WE43 exhibits no tension/compression asymmetry, weakened texture and isotropic behavior in tension.

Salient features of the fracture surfaces are summarized in Figure 5. Under uniaxial loading, Figure 5a, WE43 exhibits a mixture of trans- and intergranular fracture. Shallow dimples and ductile ridges also cover a significant portion of the fracture surface. As the triaxiality is increased, the IGF characteristics become dominant features on the fracture surface. The presence of facets and and grain pullout is easily discernible in Figs. 5b and c.

High resolution fractography of grain pull-out reveals that corners and edges of pulled out grains are decorated with second phase particles, as illustrated in Figure 6. This finding provides compelling evidence for the hypothesized role of grain boundary particles in the process of ductile intergranular failure of WE43 first shown in [12].

\section{Modeling}

\subsection{Model formulation}

A micromechanics-based framework is used to model the processes of deformation, damage initiation and accumulation to fracture. The model has three components: (i) a phenomenological law of strain-controlled void nucleation (ii) a void growth law based on porous metal plasticity that accounts for void shape effects; and (iii) an analytical shapedependent void/crack coalescence criterion.

At the material point level, additive decomposition is assumed for the total deformation rate tensor into elastic and plastic parts, $\mathbf{D}=\mathbf{D}^{\mathrm{e}}+\mathbf{D}^{\mathrm{p}}$. Assuming small elastic strains, a hypoelastic constitutive law is used for the elastic part: $\mathbf{D}^{\mathrm{e}}=\mathbb{C}^{-1}: \stackrel{\nabla}{\boldsymbol{\sigma}}$, where $\mathbb{C}$ denotes the isotropic tensor of elastic moduli and $(\nabla)$ the Jaumann rate. The plastic part of the 
deformation rate is derived from a plastic potential as follows.

$$
\mathbf{D}^{\mathrm{p}}=\Lambda \frac{\partial \Psi^{\mathrm{VG}}}{\partial \boldsymbol{\sigma}}
$$

where $\Lambda$ is the plastic multiplier and the flow potential $\Psi^{\mathrm{VG}}$ accounts for ductile damage through $[36,37]$ :

$$
\Psi^{\mathrm{VG}}\left(\boldsymbol{\sigma} ; f, W, \mathbf{e}_{3}\right)=\frac{\sigma_{\mathrm{q}}^{2}}{\bar{\sigma}^{2}}+2(g+1)(g+f) \cosh \left(\frac{3}{\kappa} \frac{\sigma_{\mathrm{h}}}{\bar{\sigma}}\right)-(g+1)^{2}-(g+f)^{2}
$$

Here, $\bar{\sigma}$ is the flow stress of the undamaged material, $f$ is the void volume fraction (also called porosity), $W$ is the void aspect ratio ( $W>1$ for elongated voids) and $\mathbf{e}_{3}$ is the void axis. The triplet $f, W$ and $\mathbf{e}_{3}$ represent internal state variables with microstructural origin. As such, they admit distributions in a real material but in a mean-field representation, averages are used. In particular, all voids are considered to be aligned with a common void axis. The stress dependence of plastic flow enters through the quadratic term involving $\sigma_{\mathrm{q}}$ and the exponential term involving $\sigma_{\mathrm{h}}$, which are defined as:

$$
\begin{aligned}
\sigma_{\mathrm{q}}^{2} & =C \frac{3}{2} \boldsymbol{\sigma}: \mathbb{H}: \boldsymbol{\sigma} \\
\sigma_{\mathrm{h}} & =\boldsymbol{\sigma}: \mathbf{X}
\end{aligned}
$$

In the original model $[36,37]$ tensor $\mathbb{H}$ contains information about the plastic anisotropy of the undamaged material, which is here neglected so that:

$$
\mathbb{H}=\mathbb{J}+\eta(\mathbf{X} \otimes \mathbf{Q}+\mathbf{Q} \otimes \mathbf{X})
$$

Here, $\mathbf{Q}$ and $\mathbf{X}$ are transversely isotropic tensors given by:

$$
\begin{gathered}
\mathbf{X} \equiv \alpha_{2}\left(\mathbf{e}_{1} \otimes \mathbf{e}_{1}+\mathbf{e}_{2} \otimes \mathbf{e}_{2}\right)+\left(1-2 \alpha_{2}\right) \mathbf{e}_{3} \otimes \mathbf{e}_{3} \\
\mathbf{Q} \equiv-\frac{1}{3}\left(\mathbf{e}_{1} \otimes \mathbf{e}_{1}+\mathbf{e}_{2} \otimes \mathbf{e}_{2}\right)+\frac{2}{3} \mathbf{e}_{3} \otimes \mathbf{e}_{3} \\
\mathbb{I}=\mathbb{I}-\frac{1}{3} \mathbf{I} \otimes \mathbf{I}
\end{gathered}
$$

where $\otimes$ stands for the dyadic product, i.e., $(\mathbf{X} \otimes \mathbf{Q})_{i j k l}=X_{i j} Q_{k l}, \mathbf{e}_{1}$ and $\mathbf{e}_{2}$ are arbitrary unit vectors forming an orthonormal triad with $\mathbf{e}_{3}, \mathbb{I}$ is the fourth-order identity tensor, $\mathbf{I}$ is the second-order identity tensor so that $\mathbb{I}$ is the deviatoric projector (for example $\mathbb{I}: \boldsymbol{\sigma}=\boldsymbol{\sigma}^{\prime}$ ). Parameters $C, g, \kappa, \eta$ and $\alpha_{2}$ are scalar valued functions of microstructural variables $f$ and $W$. For example, the expressions for $\kappa$ and $g$ are as follows.

$$
\kappa=\left\{\begin{array}{l}
\sqrt{1+\frac{1}{\ln f} \ln \frac{1-e_{2}^{2}}{1-e_{1}^{2}}} \\
{\left[1+\frac{\left(g_{f}-g_{1}\right)+\frac{4}{5}\left(g_{f}^{5 / 2}-g_{1}^{5 / 2}\right)-\frac{3}{5}\left(g_{f}^{5}-g_{1}^{5}\right)}{\ln \left(g_{f} / g_{1}\right)}\right]}
\end{array}\right.
$$




$$
g=0 \quad(\mathrm{p}) ; \quad g=f \frac{\left(1-W^{2}\right)^{\frac{3}{2}}}{W} \quad(\mathrm{o})
$$

where (p) and (o) stand for prolate and oblate voids, respectively, and $g_{f} \equiv g /(g+f)$. Other expressions can be found in Ref. [36].

To account for progressive damage by cavitation, the evolution law for the void volume fraction is written as (the superposed dot represents $\partial() / \partial t$ ):

$$
\dot{f}=(1-f) \operatorname{tr} \mathbf{D}^{\mathrm{p}}+\dot{f}_{\text {nuc }}
$$

The first term is due to void growth and comes from approximate matrix incompressibility (elastic volume change neglected). It is directly obtained from the flow potential, equation (6), using the normality property, equation (5). The second term in (14) arises from void nucleation. A strain-controlled criterion, developed by Chu and Needleman [38], is used to model void nucleation:

$$
\dot{f}_{\text {nuc }}=\mathcal{D} \dot{\bar{\epsilon}}
$$

where $\bar{\epsilon}$ is an effective matrix strain, and $\mathcal{D}$ is given by a Gaussian:

$$
\mathcal{D}(\bar{\epsilon})=\frac{f_{N}}{s_{N} \sqrt{2 \pi}} \exp \left[-\frac{1}{2}\left(\frac{\bar{\epsilon}-\epsilon_{N}}{s_{N}}\right)^{2}\right]
$$

Here, $\epsilon_{N}$ denotes an average nucleation strain, $s_{N}$ is the standard deviation and $f_{N}$ represents the volume fraction of void-nucleating particles.

The void aspect ratio is initialized upon nucleation to a certain value, $W_{0}$, then it evolves according to the micromechanical law:

$$
\begin{aligned}
\frac{\dot{W}}{W} & =\frac{3}{2} k\left[1+\left(\frac{9}{2}-\frac{T^{2}+T^{4}}{2}\right)(1-\sqrt{f})^{2} \frac{\alpha_{1}-\alpha_{1}^{\mathrm{G}}}{1-3 \alpha_{1}}\right] \mathbf{e}_{3} \cdot \mathbf{D}^{\prime \mathrm{p}} \cdot \mathbf{e}_{3} \\
& +k\left(\frac{1-3 \alpha_{1}}{f}+3 \alpha_{2}-1\right) \mathbf{I}: \mathbf{D}^{\mathrm{p}}
\end{aligned}
$$

where $T$ is the stress triaxiality ratio, $\mathbf{D}^{\prime p}$ is the deviatoric part of the plastic strain-rate, and $\alpha_{1}$ and $\alpha_{1}^{\mathrm{G}}$ are scalar valued functions of microstructural parameters $f$ and $W$; they can be found in [36]. This law, which was originally developed by Gologanu et al. [39] is modified by introducing one adjustable factor $k$.

Another key relation of the framework is

$$
(1-f) \bar{\sigma} \dot{\bar{\epsilon}}=\boldsymbol{\sigma}: \mathbf{D}^{p}
$$

where the left hand side, without the $(1-f)$, is the plastic dissipation rate per unit volume in the matrix material. This equation allows to evolve the effective plastic strain. Hardening is represented through an equation $\bar{\sigma}(\bar{\epsilon})$. During plastic loading the plastic multiplier is determined using the consistency condition, which in the actual integration of the constitutive relations, is satisfied by verifying the yield condition at the end of the increment, see [40] for further details. 
Finally, void coalescence is modeled using a recently developed yield function by Benzerga and Leblond [41] that offers a closed form solution to the problem of micro-scale localization via internal necking. The coalescence criterion reads:

$$
\begin{aligned}
\Psi^{\mathrm{VC}}(\sigma ; \chi, W) & =\frac{\left|\mathbf{e}_{3} \cdot \boldsymbol{\sigma} \cdot \mathbf{e}_{3}\right|}{\bar{\sigma}}-\frac{b}{\sqrt{3}}\left[2-\sqrt{1+3 \chi^{4}}+\ln \frac{1+\sqrt{1+3 \chi^{4}}}{3 \chi^{2}}\right] \\
& +\frac{t}{3 \sqrt{3}}\left[\frac{\chi^{3}-3 \chi+2}{\chi W}\right]
\end{aligned}
$$

where $t$ and $b$ are parameters introduced by Torki et al. [42] to improve the predictions in the case of penny-shape cracks by direct comparison with finite-element unit cell calculations. Here, $t=\left[\left(t_{0}+t_{1} \chi\right) W\right] /\left[1+\left(t_{0}+t_{1} \chi\right) W\right]$ with $t_{0}$ and $t_{1}$ as constants. To evaluate this coalescence condition, one needs to evaluate the current value of the ligament parameter $\chi$. The latter is related to $f, W$ and the void spacing ratio, $\lambda$, through:

$$
\chi=\left[\frac{3 f \lambda}{2 W}\right]^{\frac{1}{3}}
$$

The evolution of the void spacing ratio is given by:

$$
\dot{\lambda}=\frac{3}{2} \lambda D_{\mathrm{eq}}^{\mathrm{p}}
$$

where $D_{\mathrm{eq}}^{\mathrm{p}}=\sqrt{(2 / 3) \mathbf{D}^{\prime} \mathrm{p}: \mathbf{D}^{\prime} \mathrm{p}}$.

\subsection{Model implementation}

In the present work, the constitutive equations were integrated for one material point and the spatial variations of fields in the specimen were not accounted for. This method is commonly adopted when notched bars are used $[8,24]$. The above constitutive relations were integrated using a Newton-Raphson implicit scheme under the assumption of fixed void orientations. For a more general implementation suitable to finite element simulations see [40]. To apply the model, one needs to supply the initial values of the damage-relevant microstructural parameters. These consist of the void volume fraction $f$ and the void aspect ratio $W$ appearing explicitly in the void growth model, equations (12) and (13), the ligament parameter $\chi$ and, again, the void aspect ratio $W$ both appearing explicitly in the void coalescence condition (19) as well as the void spacing ratio $\lambda$ whose assumed evolution, equation (21), affects the current value of $\chi$, hence the attainment of criterion (19). The above four variables are not independent of each other; only three of them are. As noted by Pardoen and co-workers [24], the relation between them depends on the assumed unit cell. Here, the cell geometry consists of a cylindrical cell embedding a spheroidal void. Note that, to first order, the void growth stage is insensitive to the shape of the cell [21]. Also, the void coalescence condition is weakly sensitive to the shape of the void (cylindrical versus spheroidal) for a fixed set of $(W, \chi)[43]$. Thus, here $\chi$ is given by equation (20).

Furthermore, to obtain the initial relative dimensions of the hollow unit cell we proceed in three steps. First, we assume that the second phase particles are the dominant damage 
initiation sites [17], especially under triaxial loading [9]. Hence, once the nucleation criterion is met, the freshly formed voids have $\lambda_{0}=\lambda^{\text {particle }}$ and $\chi_{0}=\chi^{\text {particle }}$. On the other hand, $W_{0}$ is taken to be different from $W^{\text {particle }}$ as the incipient voids have been observed to nucleate as penny-shaped cracks either due to particle cracking or decohesion at the particle/matrix interface; see [24] for similar reasoning. It is worth mentioning that the results are nearly insensitive to the specific value of $W_{0}$ when $W_{0} \leqslant 0.1$. This was first noted by Scheyvearts et al. [44] for flat voids in isotropic matrices and further confirmed by Keralavarma et al. [45] in the case of anisotropic matrices.

Next, not only the cylindrical cell is not space filling but also void dimensions and void spacings in the plane of coalescence are direction dependent. Since this cannot be represented using an axisymmetric cell, an intermediate orthorombic cell is defined based on measured mean void spacings (Figure 7). Then the passage from this intermediate to the cylindrical cell is made by using geometric means of dimensions in the plane of coalescence [23]. Accordingly, the microstructural features are defined as follows.

$$
f=\frac{V_{\text {void }}}{V_{\text {cell }}} ; \quad W=\frac{h}{d} ; \quad \lambda=\frac{H}{R} ; \quad \chi=\frac{d}{R}
$$

where $V_{V o i d}$ and $V_{\text {Cell }}$ are the volume of one of the voids and volume of the cell, respectively. The parameter $h$ is the height of the void, $d$ is the average of the void diameters along $\mathrm{S}$ and T directions, i.e., $d=\sqrt{d_{S} d_{T}}, H$ is the cell's height and $R$ is the mean width of the cell, defined as $R=\sqrt{R_{S} R_{T}}$.

Finally, since IGF is prominent in this alloy, we assume that the process is mediated by grain-boundary particles, which therefore require special attention. The microstructure of the alloy should be idealized based on IGF and grain boundary particles. Figure 8 presents an example of such idealization for WE43 in $\mathrm{L}-\mathrm{T}$ plane. Since a certain degree of variation in particle diameters and their spacing is expected in real materials, various strategies are used to calculate the parameters of the representative cell in Figure 8. Following Pardoen et al. [24], we determine $\chi_{0}$ and $\lambda_{0}$ based on the location of grain boundary particles.

The plastic strain increment is calculated using equation (5) and the change in microstructural variables are measured using evolution laws. The fracture is defined by the onset of micro-scale localization (coalescence), characterized by meeting the coalescence condition, equation (19), before void growth, equation (6). An equivalent measure of the accumulated strain is defined as

$$
\bar{\varepsilon}=\sqrt{\frac{2}{3} \varepsilon^{\prime}: \varepsilon^{\prime}} \quad \text { with } \quad \varepsilon=\int_{0}^{t} \mathbf{D} d t
$$

and the fracture strain is taken to be its value when the coalescence criterion is satisfied.

\subsection{Material Parameter Identification}

Three types of parameters are involved in the model: (i) the initial values of damagerelevant microstructural parameters, namely $W_{0}, \chi_{0}$ and $\lambda_{0}$; (ii) the constitutive parameters of the dense, undamaged matrix, including elastic constants $E$ and $\nu$, initial strength and hardening parameters; and (iii) the adjustable parameters $k$ in equation (17) and $t$ and $b$ in the coalescence criterion (19). 
The void nucleation is modeled according to equation (16), where choosing $\varepsilon_{N}=0$ and $s_{N}=0$ represents a matrix with pre-existing voids, $f_{0}$, the value of which is calculated later and no nucleation of new void during plastic deformation. This is based on the fact that brittle second phase particles are prone to nucleate voids after limited plastic deformation [46].

Because of the insensitivity of results to the value of $W_{0}$ below a certain threshold, the value of 0.05 was assumed for incipient crack-like voids. As stated above, determination of $\chi_{0}$ and $\lambda_{0}$ requires that the dimensions of incipient voids and their inter-spacings be measured. This was accomplished using image analysis and quantitative metallography based on two dimensional micrographs in $\mathrm{L}-\mathrm{T}$ plane and $\mathrm{L}-\mathrm{S}$ plane. The average particle diameter and mean center-to-center particle spacing along principal directions for particles on grain boundaries are presented in Table 2. No streology is used to calculate the $3 \mathrm{D}$ values of of $d$ and $R$. Instead, $\chi_{0}$ is calculated based on equation 22 using $d$ and $R$, which are obtained via geometric averaging of $d_{T}, d_{S}, R_{T}$ and $R_{S}$ as discussed in Section 4.2. The parameter $\lambda_{0}$ is calculated as the ratio of the average center-to-center particle spacing, $H=4.7 \mu \mathrm{m}$, to $R$. More sophisticated approaches require recourse to stereological relationships [26]. Following this procedure, these values were arrived at: $\lambda_{0}=1.77$ and $\chi_{0}=0.22$. Along with $W_{0}=$ 0.05 , these values lead to an estimated initial void volume fraction of $f_{0}=0.0002$. These are nominal values but to account for various sources of uncertainty in material parameter identification, parameter sensitivity analyses were carried out using the model. Results showed that among all microstructural variables, the model is most sensitive to variation of $\chi_{0}$. Thus, two extreme values of $\chi_{0}=0.17$ and $\chi_{0}=0.31$ were used to identify the uncertainty due to material parameter identification. The two extreme values of $\chi_{0}$ are obtained from averaging the ligament ratio of individual particles over all studied particles in $\mathrm{L}-\mathrm{T}$ and $\mathrm{L}-\mathrm{S}$ plane.

The constitutive parameters of the undamaged matrix include Young's modulus, $E=$ $44 \mathrm{GPa}$, Poisson's ratio, $\nu=0.27$ and a hardening law written in the form:

$$
\bar{\sigma}=K(0.002+\bar{\epsilon})^{n}
$$

where $K=615.0 \mathrm{MPa}$ and $n=0.16$ are determined based on the best fit to the true stress-strain curve in tension along the L direction, Figure 3a. A summary of the material parameters with qualitative description of their effect on the overall ductility is presented in Table 3.

Regarding the heuristic parameters entering the model, parameters $b, t_{0}$ and $t_{1}$ were calibrated by Torki et al. [42] based on unit cell calculations. Their values of $b=0.9$, $t_{0}=-0.84$ and $t_{1}=12.9$ are used here. Thus, the only adjustable parameter is $k$ which enables to modulate the effect of blunting of crack-like defects. In the original model $k=1$. Any value below 1 means that the crack-like voids do not open as quickly as in the $k=1$ case.

\subsection{Model Predictions}

Figure 9 compares the experimentally measured fracture locus for loading along the rolling, (L), direction with two sets of predictions corresponding to two values of parameter $k$, 
introduced in equation (17). When $k=1$, as in the original void growth equations, the model is unable to predict the experimentally measured values of strain to failure. On the other hand, the value of $k=0.43$ offers very close predictions to the experimental values. Using the range of uncertainties associated with $\chi_{0}$, identified in Section 4.3, a prediction range for the model is obtained for the case of $k=0.43$. The predicted ranges of fracture strains encompass the available experimental data. It is quite remarkable that such a simple heuristic modification to the evolution equation of the void aspect ratio can have such incidence on the predicted fracture strains. This will be discussed further below.

Recall that the predicted fracture strains are for prescribed proportional stressing paths with triaxialities as indicated in the abscissa of Figure 9. The predicted fracture strains are actually the result of a continuous damage accumulation process. To appreciate this, Figure 10 shows some typical effective stress-strain responses as well as the evolution of internal variables up to the onset of fracture, which is indicated by the symbol ' $x$ '. The onset of fracture is defined by meeting the coalescence condition, equation (19) before (6). This depends on the current values of the void aspect ratio and the ligament parameter, the evolution of which are shown in Figure $10 \mathrm{~b}$ and c, respectively. Prior to plastic flow, the internal variables remain constant (for strains below 0.005 or so). Due to the low value of $k$, crack-like void opening is slowed down and at coalescence the voids have $W_{c}<0.2$. Such low values do not explain per se the relatively low fracture strains predicted in that case. If anything, all things kept fixed, a low value of $W$ makes the coalescence condition harder to meet as the coalescence stress scales inversely with $W$. However, constraining $W$ to evolve slowly and remain close to crack-like voids leads to a faster evolution of the ligament parameter by virtue of equation (20). Ultimately, higher values of $\chi$ promote void coalescence. Here, the values of $\chi_{c}$ at coalescence are predicted to be in the range 0.3 to 0.5 , which is the usual range regardless of initial conditions; see [47].

What is remarkable in Figure 10a is that fracture sets in without any prior softening. Such qualitative behavior would be rather difficult to simulate, if at all possible, using isotropic damage models, such as the Gurson model. To understand this, recall that fracture is defined by the onset of coalescence. In this regard, the attainment of condition (19) is not contingent upon there being any softening in the material response, even at the local level. Finally, note that the plasticity model assumed in the matrix only depends on deviatoric stresses so that the triaxiality has no effect on the plastic response of the matrix. Due to the low porosity values encountered such triaxiality-independence of the stress strain curve translates to the effective behavior. The small effect seen in Figure 10a is due to the fact that porosity increase is faster at higher triaxiality, as shown in Figure 10d. Also note that the porosity levels at the onset of fracture are so low they would be barely discernable on transverse fractographs in interrupted tests.

\section{Discussion}

The modeling strategy adopted in this work follows the methodology put forth by Benzerga et al. [23] for modeling anisotropic damage accumulation to fracture with notable differences. On the one hand, the constitutive relations employed here are based on more recent developments of anisotropic void growth [36,37] and void coalescence [41,42]. Note that 
because of the weak plastic anisotropy of WE43 alloy, the void growth model used here reduces to one which is quite similar to the model used in [23] originally developed by Gologanu et al. [39]. On the other hand, this modeling approach is implemented here in a simplified way. Unlike in [23] where full boundary value problem solutions were obtained to simulate crack growth and propagation in notched bars, here proportional stressing paths were simulated and assumed to mimic those encountered at failure locations in actual specimens. This obviously entails approximations. Analysis by Pineau and co-workers [8] shows that during deformation of notched bars, the triaxiality remains roughly constant at fracture location, making these specimens ideal "constant triaxiality" specimens provided that notch integrity is not lost. Finite element calculations show that this is the case for a quasi-isotropic material for strains below 0.3 [23]. The representation of the initial damagerelevant microstructure followed the approach proposed by Pardoen et al. [24] to model intergranular versus transgranular fracture in aluminum alloys. This approach is based on the concept of a unit cell to account for the peculiar spatial distribution of second-phase particles associated with the observed intergranular fracture mechanism. It begins with an idealization of the damage-relevant microstructure, as illustrated in Figure 8, in order to define a unit cell for implementing the micromechanical damage model.

Combining our experimental observations with the results of micromechanical modeling enables to rationalize the low fracture strains in WE43 alloy compared with AZ31 for triaxial loadings (Figure 4). In light of the present findings, the fracture process in WE43 may be explained as follows. After a critical strain, which is probably negligibly small, crack-like voids nucleate at particles located on suitably oriented grain boundaries. For reasons yet to be fully elucidated, the blunting of crack-like voids is slower than predicted by continuum theory. This aspect is accounted for in the model via the heuristic factor $k$. Hence, void growth is truncated soon after nucleation due to the spatial arrangement of grainboundary particles, which is favorable to early commencement of coalescence. The seemingly brittle intergranular fracture is still a ductile fracture process that can be represented using the anisotropic models implemented here. By way of consequence, triaxiality effects still manifest, as shown both experimentally and computationally in Figure 9. Under uniaxial tension, possible interference from shear localization reduces the strain that the alloy can accommodate before final failure and leads to a maximum in fracture locus at moderate triaxialities, similar to the case of AZ31 [9].

Clearly, the intergranular character of fracture originates from the high number density of second phase particles on grain boundaries. Therefore, removing precipitates and second phase particles from these boundaries can be effective in improving ductility. Unfortunately, because of the strong propensity of RE elements to segregate at boundaries, removing second phase particles from them is challenging. It is unclear whether heat treatments can dissolve all grain boundary precipitates in RE-concentrated alloys. In fact, Hadorn et al. [5] showed that annealing at $673 \mathrm{~K}$ does not "desegregate" $\mathrm{RE}$ elements from grain boundaries. On the other hand, second phase precipitates and particles participate in texture weakening of Mg-RE elements by retarding the dynamic recrystalization through Zener pinning effect. Solute segregation also reduces the boundary movement in Mg-RE alloys. Removing these particles and segregated atom from boundaries, if at all possible, requires high temperature thermo-mechanical processes. In that case, the properties of processed material, including re-crystallized texture and strength, can be significantly different from those processed at 
lower temperatures. Recently, Kumar et al. [30] showed that friction stir processing can effectively increase the ductility of WE43 sheets by physically moving second phase particles from grain boundaries to the grain interior.

The proposed model relies on one adjustable parameter, $k$ in equation (17). It is remarkable that such a simple heuristic amendment to the void shape evolution can have such impact on the results (see Figure 9). To gain further insight into this, Figure 11 shows the stress-strain response along with the evolution of damage-relevant microstructural variables for two values of $k$ in the $T=1.45$ case using $\chi_{0}=0.22$. The effect of varying $k$ is barely visible on the stress strain curve (Figure 11a) or the rate of growth of void volume fraction (Figure 11d). In fact, no porosity measurement could discriminate among the two cases. However, the effect of $k$ on the evolution of the void aspect ratio (Figure 11b) and the ligament parameter (Figure 11c) is quite clear. Using a lower value for $k$ slows down void opening, hence the rate of $W$. As a consequence, the ligament parameter $\chi$ evolves much faster by virtue of equation (20) and hence reaches the critical range for coalescence earlier than in the $k=1$ case. We emphasize that since the evolution of porosity is the same before fracture, no isotropic model would be able to distinguish among the two situations in Figure 11. This shows that anisotropic damage models are necessary for modeling fracture in materials failing after limited void growth by the coalescence of crack-like voids.

The material in the current calculation is assumed to contain pre-existing voids. This is a reasonable assumption considering the fact that brittle second phase particles in $\mathrm{Mg}$ are susceptible to breaking after limited plastic strain. This premise is also based on metallographic observations in hot-rolled AZ31 that shows most second phase particles are broken at incipient plasticity. Despite physical evidence, this assumption may lead to errors and is not necessarily the most accurate assumption [38]. Lower scale calculation of twin- and particle-induced cracks/voids can be instrumental in developing more sophisticated criteria for void nucleation in $\mathrm{Mg}$ alloys. At this stage, however, scarcity of data in the literature justifies the assumption of pre-existing voids.

Further research is needed to corroborate the present findings. For instance, it remains to be seen whether the model can predict triaxiality-dependent fracture in AZ31 alloy. In general, this would require using an anisotropic plasticity model in the matrix that eventually accounts for tension-compression asymmetry. It is also relevant to reflect on the physical implications of $k$ being significantly smaller than unity. First, it is important to note that the value used is more consistent with qualitative microscopy observations which do suggest limited micro-crack blunting, definitely less than predicted based on continuum theory [36] or cell model calculations [44]. Several factors could be studied to rationalize the limited void opening. First, at the scale of the voids plastic flow is anisotropic to account for crystallographic aspects. Locally anisotropic behavior may hinder void opening. However, the cell model calculations in [45], although not exhaustive, suggest that this may not be sufficient explanation. Another aspect is the particle locking effect [8], as modeled for example in [48], which manifests when nucleation occurs by particle cracking. However, such phenomenon is expected to diminish at moderate to high triaxiality. Finally, it is also possible that length scale effects play a role. For instance, when crack-like voids form one dimension, the crack height, is definitely in the sub-micron scale so that application of continuum plasticity becomes questionable. If dislocation free zones develop above and below the crack-like void, it may be difficult to extend the crack in the direction of the loading. To 
address this, lower scale analyses, such as discrete dislocation plasticity, are needed.

\section{Conclusion}

The process of plasticity induced damage accumulation to fracture in a magnesium WE43 alloy was investigated. The main findings are as follows.

- Experiments show that WE43 does not exhibit tension-compression asymmetry and is nominally isotropic. Fractography indicates that intergranular fracture controls ductility. High resolution fractographs illustrate that second phase particles, decorating the grain boundaries, play an important role in the process of damage and fracture of WE43.

- A micromechanics-based continuum damage model is employed to rationalize the key experimental trends. This model contains a phenomenological law of strain-controlled void nucleation, a void growth part that accounts for microcrack blunting and a void coalescence part that determines fracture.

- A systematic method for identifying the material parameters is presented. In addition, using a parameter sensitivity analysis, a range of material parameters are identified to capture the effect of uncertainties in the material parameter identification procedure.

- Using material parameters associated with grain-boundary particles and employing a single heuristic parameter that governs the rate of microcrack blunting, the model predictions are found to lie very close to experimentally obtained strains to fracture.

- Model predictions show that crack-like voids on grain boundaries coalesce after limited straining. This early coalescence is attributed to the slow blunting of the crack-like voids.

More generally, it is demonstrated that the micromechanical model, which is usually used to describe dimpled fracture, can be used to model intergranular fracture. This is rooted in the model's ability to account for void shape effects on both growth and coalescence in order to describe penny-shape cracks as essential damage entities.

\section{Acknowledgments}

The research reported herein was conducted under the support of NPRP grant No 4-1411-2555 from the Qatar National Research Fund (a member of Qatar Foundation). The authors thank Magnesium Elektron for supplying the material. AAB also acknowledges partial support from the National Science Foundation under Grant number CMMI-1405226. 


\section{References}

[1] B. C. Wonsiewicz, B. W. A., Plasticity of magnesium crystals, Trans. TMS-AIME 239 (1967) 1422-1431.

[2] S. R. Agnew, J. F. Nie, Preface to the viewpoint set on: The current state of magnesium alloy science and technology, Scripta Materialia 63 (2010) 671-673.

[3] N. Stanford, G. Sha, J. H. Xia, S. P. Ringer, M. R. Barnett, Solute segregation and texture modification in an extruded magnesium alloy containing gadolinium, Scripta Materialia 65 (2011) 919-921.

[4] S. Sandloebes, S. Zaefferer, I. Schestakow, S. Yi, R. Gonzalez-Martinez, On the role of non-basal deformation mechanisms for the ductility of $\mathrm{Mg}$ and $\mathrm{Mg}-\mathrm{Y}$ alloys, Acta Materialia 59 (2011) 429-439.

[5] J. P. Hadorn, K. Hantzsche, S. Yi, J. Bohlen, D. Letzig, J. A. Wollmershauser, S. R. Agnew, Role of solute in the texture modification during hot deformation of Mg-rare earth alloys, Metallurgical and Materials Transactions A. 43 (2012) 1347-1362.

[6] S. Sandloebes, Z. Pei, M. Friak, L.-F. Zhu, F. Wang, S. Zaefferer, D. Raabe, J. Neugebauer, Ductility improvement of Mg alloys by solid solution: Ab initio modeling, synthesis and mechanical properties, Acta Materialia 70 (2014) 92-104.

[7] Z. Leng, J. Zhang, J. Sun, H. Shi, S. Liu, L. Zhang, M. Zhang, R. Wu, Notch tensile behavior of extruded Mg-Y-Zn alloys containing long period stacking ordered phase, Materials \& Design 600 (2014) 495-499.

[8] A. Pineau, A. Benzerga, T. Pardoen, Failure of metals I: Brittle and ductile fracture, Acta Materialia 107 (2016) 424-483.

[9] B. Kondori, A. A. Benzerga, Effect of stress triaxiality on the flow and fracture of $\mathrm{Mg}$ alloy AZ31, Metallurgical and Materials Transactions A. 45 (2014) 3292-3307.

[10] B. Kondori, A. A. Benzerga, Fracture strains, damage mechanisms and anisotropy in a magnesium alloy across a range of stress triaxialities, Experimental Mechanics 54 (2014) 493-499.

[11] B. Selvarajou, B. Kondori, A. A. Benzerga, S. P. Joshi, On plastic flow in notched hexagonal close packed single crystals, Journal of the Mechanics and Physics of Solids 94 (2016) 273-297.

[12] B. Kondori, A. A. Benzerga, On the notch ductility of a magnesium-rare earth alloy, Materials Science and Engineering:A 647 (2015) 74-83.

[13] T. E. Davidson, J. C. Uy, A. P. Lee, Tensile fracture characterization of metals under hydrostatic pressures to 23 kilobars, Acta Metallurgica 14 (1966) 937-948.

[14] M. R. Barnett, Twinning and the ductility of magnesium alloys Part II. "contraction" twins, Materials Science and Engineering:A 464 (2007) 8-16. 
[15] D. Ando, J. Koike, Y. Sutou, The role of deformation twinning in the fracture behavior and mechanism of basal textured magnesium alloys, Materials Science and Engineering:A 600 (2014) 145-152.

[16] M. Lentz, M. Risse, N. Schaefer, W. Reimers, I. Beyerlein, Strength and ductility with $\{10 \overline{1} 1\}-\{10 \overline{1} 2\}$ double twinning in a magnesium alloy, Nature Communications 7 (2016) 11068.

[17] M. Lugo, M. A. Tschopp, J. B. Jordon, M. F. Horstemeyer, Microstructure and damage evolution during tensile loading in a wrought magnesium alloy, Scripta Materialia 64 (2011) 912-915.

[18] S. Lee, S. Lee, D. Kim, Effect of Y, Sr, and Nd additions on the microstructure and microfracture mechanism of squeeze-cast AZ91-X magnesium alloys, Metallurgical and Materials Transactions A. 527 (1998) 1221-1235.

[19] B. Kim, J. Do, S. Lee, I. Park, In situ fracture observation and fracture toughness analysis of squeeze cast AZ51-xSn magnesium alloys, Materials Science and Engineering:A 527 (2010) 6745-6757.

[20] D. Steglich, T. F. Morgeneyer, Failure of magnesium sheets under monotonic loading: 3d examination of fracture mode and mechanisms, International Journal of Fracture 183 (2013) 105-112.

[21] A. A. Benzerga, J.-B. Leblond, Ductile fracture by void growth to coalescence, Advances in Applied Mechanics 44 (2010) 169-305.

[22] A. A. Benzerga, J.-B. Leblond, A. Needleman, V. Tvergaard, Ductile Failure Modeling, International Journal of Fracture 201 (2016) 29-80.

[23] A. A. Benzerga, J. Besson, A. Pineau, Anisotropic ductile fracture. Part II: Theory, Acta Materialia 52 (2004) 4639-4650.

[24] T. Pardoen, D. Dumont, A. Deschamps, Y. Brechet, Grain boundary versus transgranular ductile failure, Journal of the Mechanics and Physics of Solids 51 (2003) $637-665$.

[25] R. T. DeHoff, Measurement of number and average size in volume, in: R. T. DeHoff, F. N. Rhines (Eds.), Quantitative Microscopy, McGraw-Hill, New York, 1968, pp. 128148.

[26] A. A. Benzerga, J. Besson, A. Pineau, Anisotropic ductile fracture. Part I: Experiments, Acta Materialia 52 (2004) 4623-4638.

[27] R. Xin, B. Song, K. Zeng, G. Huang, Q. Liu, Effect of aging precipitation on mechanical anisotropy of an extruded Mg-Y-Nd alloy, Materials \& Design 34 (2012) 384-388.

[28] K. Hantzsche, J. Bohlen, J. Wendt, K. U. Kainer, S. B. Yi, D. Letzig, Effect of rare earth additions on microstructure and texture development of magnesium alloy sheets, Scripta Materialia 63 (2010) 725-730. 
[29] J. Bohlen, M. R. Nuernberg, J. W. Senn, D. Letzig, S. R. Agnew, The texture and anisotropy of magnesium-zinc-rare earth alloy sheets, Acta Materialia 55 (2007) 21012112 .

[30] N. Kumar, N. Dendge, R. Banerjee, R. S. Mishra, Effect of microstructure on the uniaxial tensile deformation behavior of Mg-4Y-3RE alloy, Materials Science and Engineering:A 590 (2014) 116-131.

[31] I.-B. Kim, J.-Y. Hong, B.-G. Hong, K.-H. Kim, Precipitation behavior of Mg-Y-Nd-Zr alloy, Materials Science Forum 449-452 (2004) 649-652.

[32] S. Delfino, A. Saccone, R. Ferro, Phase relationships in the neodymium-magnesium alloy system, Metallurgical Transactions 21 (1990) 2109-2114.

[33] J. Grobner, R. Schmid-Fetzer, Thermodynamic modeling of the Mg-Ce-Gd-Y system, Scripta Materialia 63 (2010) 674-679.

[34] F. G. Meng, J. Wang, H. S. Liu, L. B. Liu, Z. P. Jin, Experimental investigation and thermodynamic calculation of phase relations in the $\mathrm{Mg}-\mathrm{Nd}-\mathrm{Y}$ ternary system, Materials Science and Engineering:A 454-455 (2007) 266-273.

[35] A. Kielbus, M. Stopyra, R. Jarosz, Influence of sand-casting parameters on microstructure and properties of magnesium alloys, Archives of Metallurgy and Materials 58 (2013) 635-640.

[36] S. M. Keralavarma, A. A. Benzerga, A constitutive model for plastically anisotropic solids with non-spherical voids, Journal of the Mechanics and Physics of Solids 58 (2010) 874-901.

[37] S. Keralavarma, A. Benzerga, Numerical assessment of an anisotropic porous metal plasticity model, Mechanics of Materials 90 (2015) 212-228.

[38] C. Chu, A. Needleman, Void nucleation effects in biaxially stretched sheets, Journal of Engineering Materials and Technology 102 (1980) 249-256.

[39] M. Gologanu, J.-B. Leblond, G. Perrin, J. Devaux, Recent extensions of Gurson's model for porous ductile metals. Part I: Gurson-like models incorporating the influence of void shape, in: P. Suquet (Ed.), Continuum Micromechanics, Springer, Verlag, 1995, pp. $61-97$.

[40] S. Kweon, B. Sagsoy, A. Benzerga, Constitutive relations and their time integration for anisotropic elasto-plastic porous materials, Computer Methods in Applied Mechanics and Engineering (2016) -Article in Press, doi:http://dx.doi.org/10.1016/j.cma.2016.06.005.

[41] A. A. Benzerga, J.-B. Leblond, Effective yield criterion accounting for microvoid coalescence, Journal of Applied Mechanics 81 (2014) 1-7. 
[42] M. E. Torki, A. A. Benzerga, J.-B. Leblond, On Void Coalescence under Combined Tension and Shear, Journal of Applied Mechanics 82 (7) (2015) 071005.

[43] L. Morin, J.-B. Leblond, A. Benzerga, D. Kondo, A unified criterion for the growth and coalescence of microvoids, Journal of the Mechanics and Physics of Solids (2016) -Article in Press, doi:http://dx.doi.org10.1016/j.jmps.2016.01.013.

[44] F. Scheyvaerts, T. Pardoen, P. Onck, A new model for void coalescence by internal necking, International Journal of Damage Mechanics 19 (2010) 95-126.

[45] S. M. Keralavarma, S. Hoelscher, A. A. Benzerga, Void growth and coalescence in anisotropic plastic solids, International Journal of Solids and Structures 48 (2011) 16961710.

[46] M. Kikuchi, K. Shiozawa, J. Weertman, Void nucleation in astroloy: theory and experiments, Acta Metallurgica 29 (1981) 1747-1758.

[47] A. A. Benzerga, Micromechanics of coalescence in ductile fracture, Journal of the Mechanics and Physics of Solids 50 (2002) 1331-1362.

[48] K. Siruguet, J.-B. Leblond, Effect of void locking by inclusions upon the plastic behavior of porous ductile solids-I: Theoretical modeling and numerical study of void growth, International Journal of Plasticity 20 (2004) 225-254.

[49] Magnesium Elektron Ltd, Elektron43 preliminary datasheet, URL: http://www.magnesium-elektron.com/data/downloads/DS043.pdf, Magnesium Elektron Ltd, Swinton, England, Accessed: 09/15/2014 (2011). 
Table 1: Nominal chemical composition of WE43 alloy; from [49].

\begin{tabular}{|l||c|c|c|c|}
\hline Alloy / Element & Yttrium & Rare Earth & Zirconium & Magnesium \\
\hline WE43 & $3.7-4.3(\%)$ & $2.3-3.5(\%)$ & $0.2(\%)$ min. & bal. \\
\hline
\end{tabular}


(a)

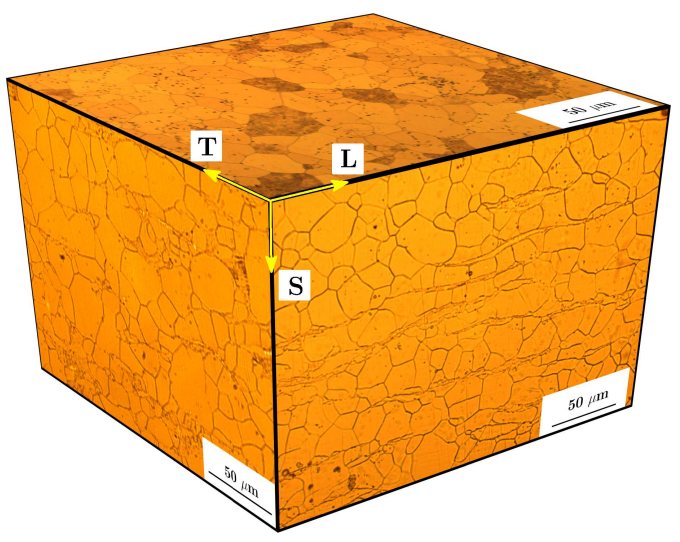

(b)

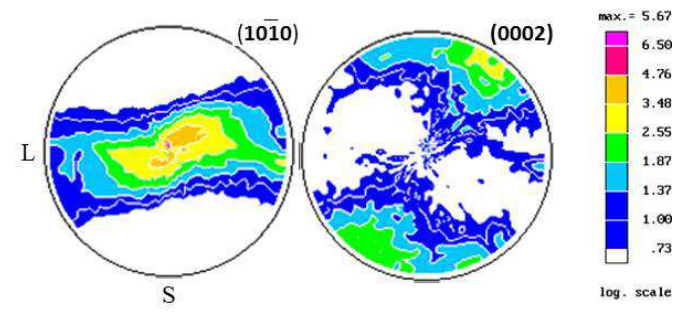

Figure 1: (a) 3D optical microscope representation of initial microstructure of WE43 hotrolled plate. (b) XRD pole figures corresponding to (1010) and (0002) planes. 


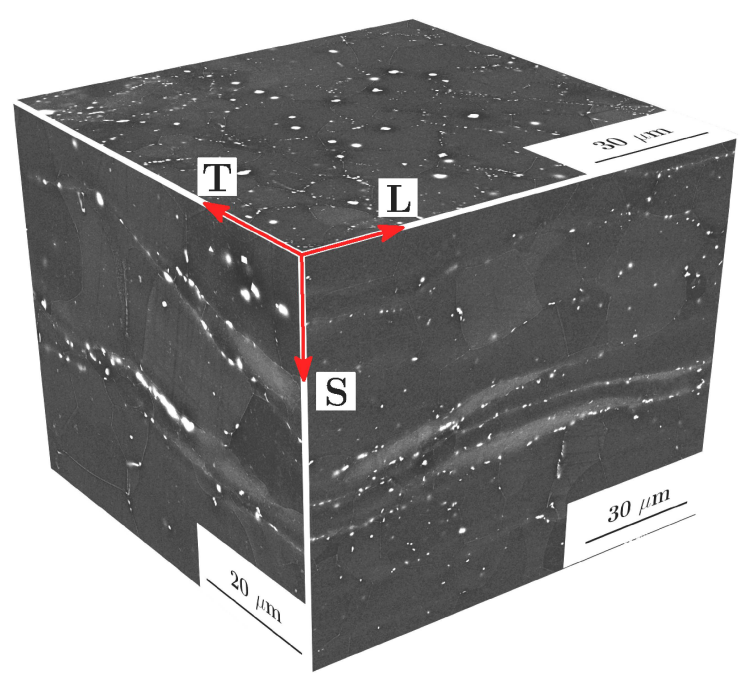

Figure 2: 3D SEM representation of WE43 initial microstructure showing the spatial distribution of second phase particles. 
(a)
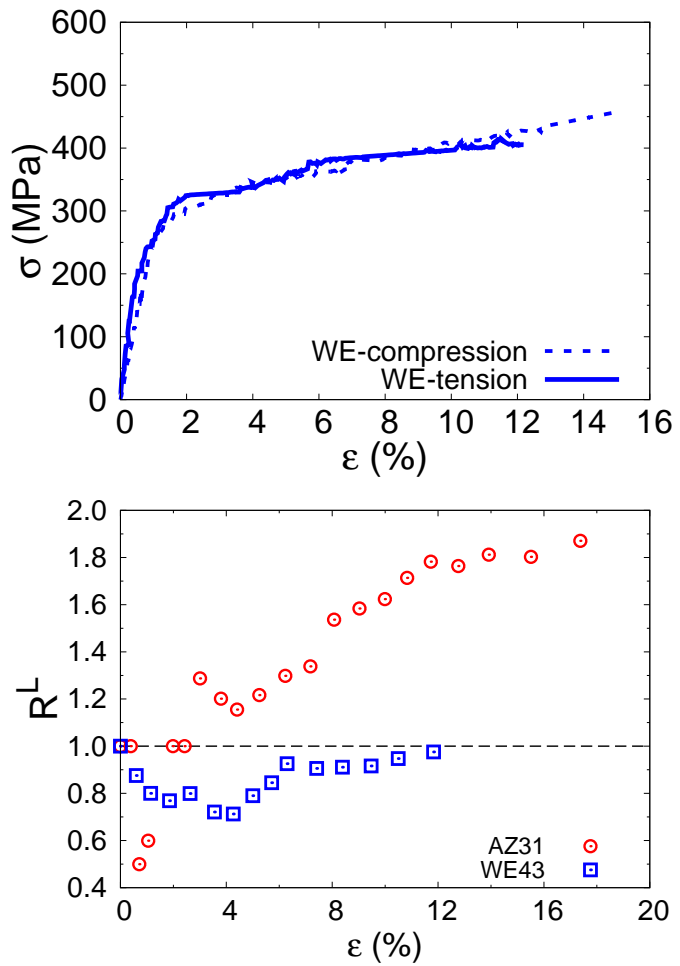

Figure 3: True stress versus true strain response of WE43 tested along the L orientation in tension and compression. (b) Evolution with plastic strain of the anisotropy ratio, $R^{L}$, in tension of WE43 and AZ31 alloys. 


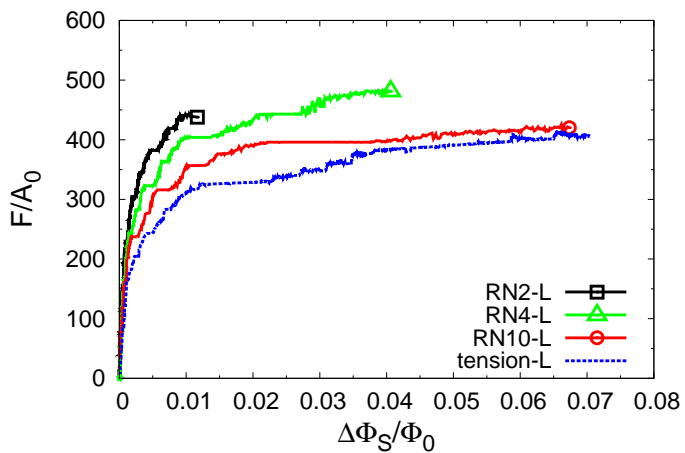

(a)

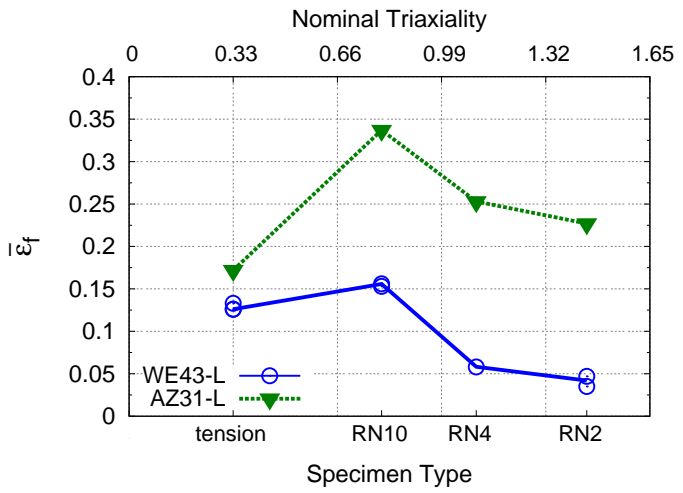

Figure 4: (a) Normalized force versus diameter reduction along the $\mathrm{S}$ direction for the uniaxial and notched tensile specimens. (b) Fracture loci of WE43 and AZ31 alloys. 


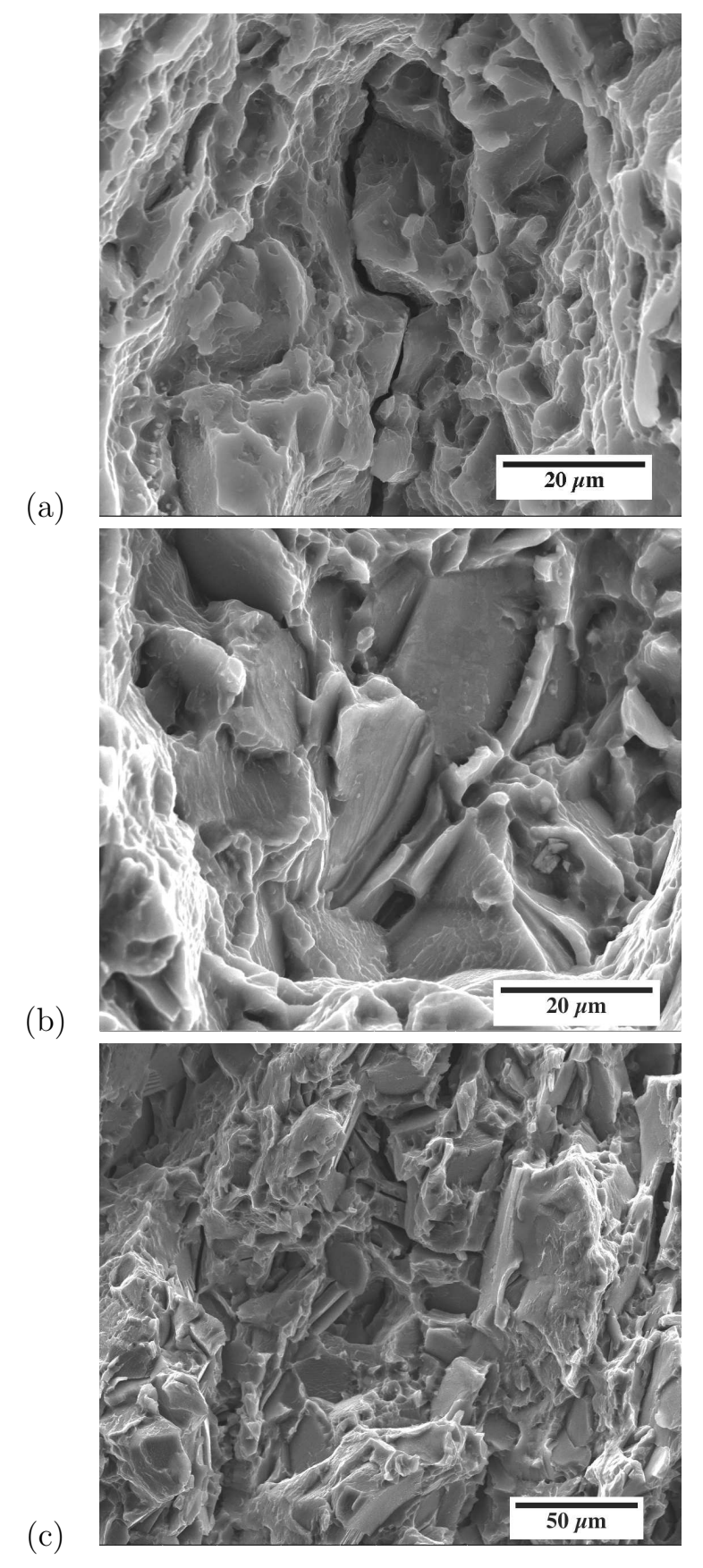

Figure 5: Fracture surfaces of (a) a tensile bar; (b) RN10 bar; and (c) RN2 bar. 


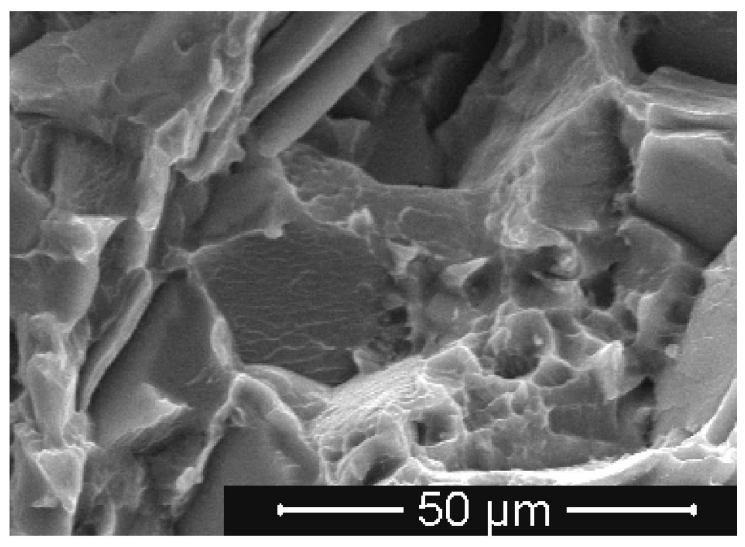

Figure 6: Detail on the fracture surface of an RN2 specimen showing a grain at its center. The boundary of this grain is decorated with second phase particles suggesting their role in intergranular fracture. 


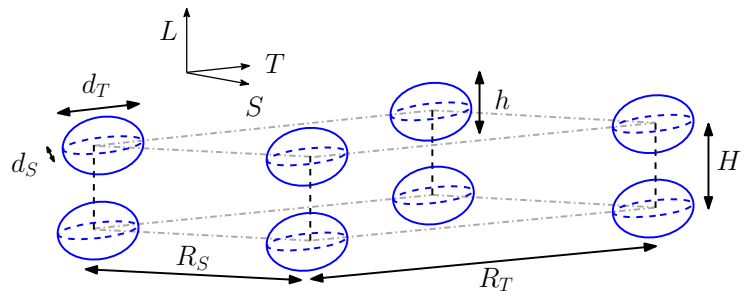

Figure 7: Representative volume element for a material containing voids represented as 3D ellipsoids centered on the vertices of a tetragonal cell. 


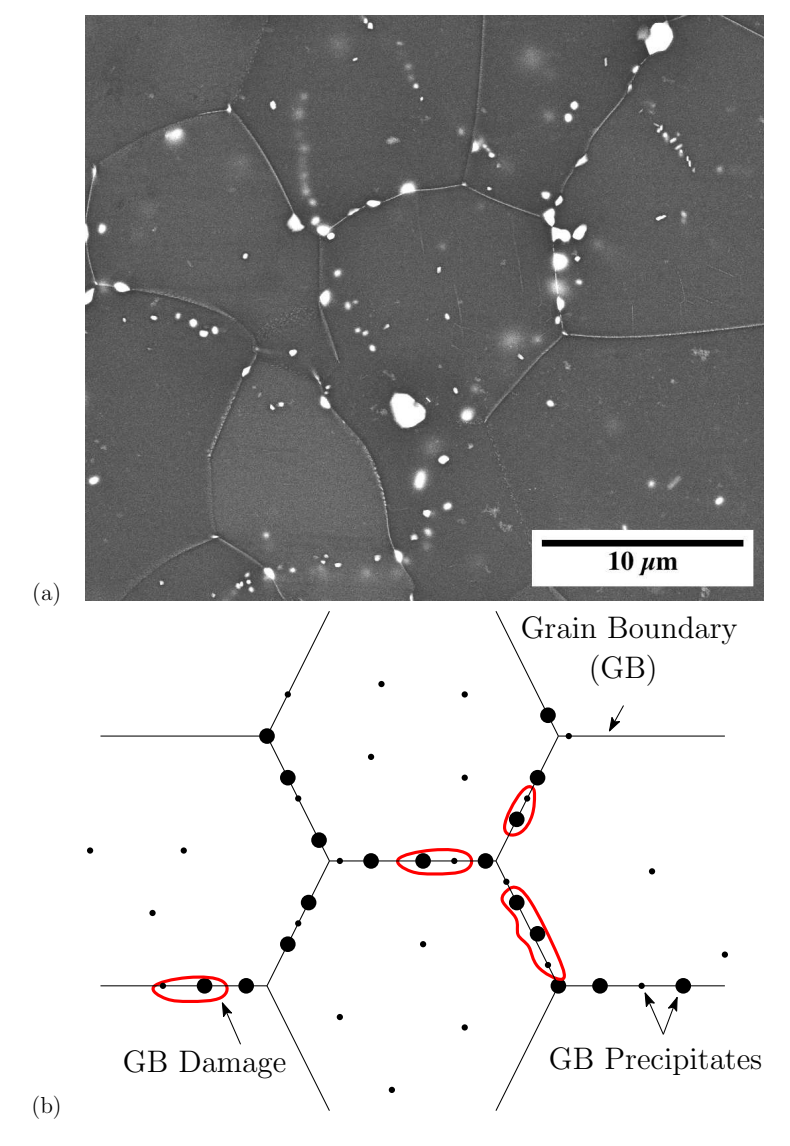

Figure 8: (a) Microstructure of WE43 in L-T plane showing second phase particles located on or near grain boundaries. (b) Schematic of the idealized microstructure for study of intergranular fracture using models for ductile fracture. 
Table 2: The average particle diameter, $d$, and mean particle distance, $R$, on all grain boundaries along principal directions.

\begin{tabular}{|l|l|l|}
\hline direction & diameter $(\mu \mathrm{m})$ & spacing $(\mu \mathrm{m})$ \\
\hline $\mathrm{L}$ & $h=0.4$ & $H=4.7$ \\
\hline $\mathrm{T}$ & $d_{T}=0.6$ & $R_{T}=2.5$ \\
\hline $\mathrm{S}$ & $d_{S}=0.6$ & $R_{S}=2.8$ \\
\hline
\end{tabular}


Table 3: Initial values of microstructural parameters and other parameters used in the calculations.

\begin{tabular}{|c|c|c|c|}
\hline Symbol & Physical meaning & Initial Value (range) & Sensitivity \\
\hline$f$ & void volume fraction & 0.0002 & small \\
\hline$W$ & void shape & 0.05 & small \\
\hline$\lambda$ & relative void spacing ratio & 1.77 & large \\
\hline$\chi$ & ligament ratio & $0.22(0.17 \leqslant \chi \leqslant 0.31)$ & large \\
\hline$n$ & hardening exponent & 0.16 & small \\
\hline$E$ & Elastic Modulus & $440 \mathrm{GPa}$ & small \\
\hline$\nu$ & Poisson's ratio & 0.27 & small \\
\hline
\end{tabular}




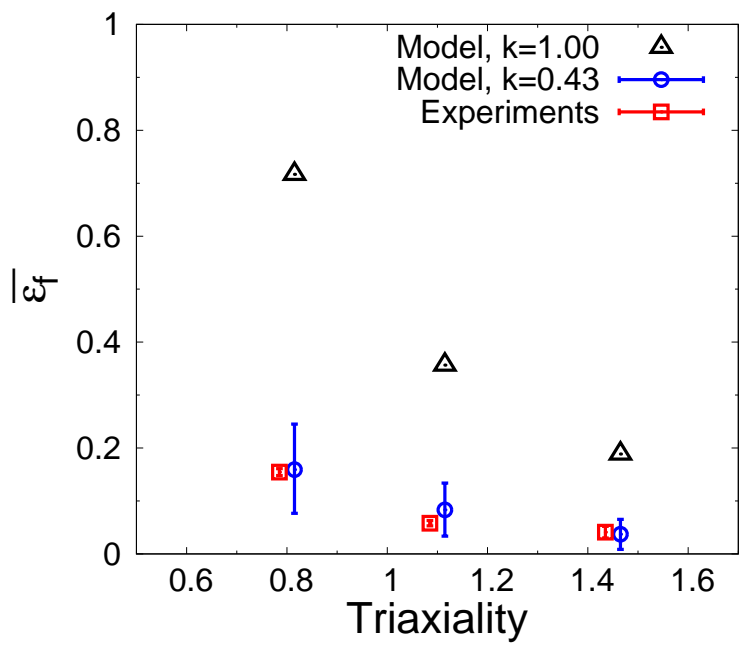

Figure 9: Comparison between predicted and experimentally measured fracture strains in WE43 loaded parallel to the L direction. Two sets of predictions are related to two values of parameter $k$ in equation (17). The scatter bar in the predicted fracture strains corresponds to different values of the ligament parameter $\chi$. 
(a)

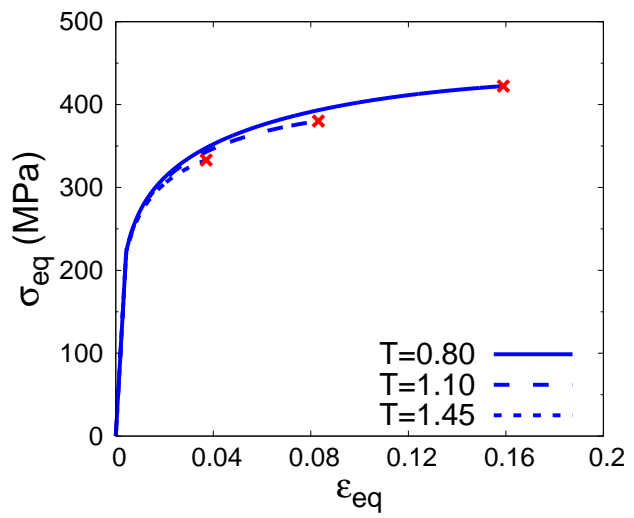

(c)

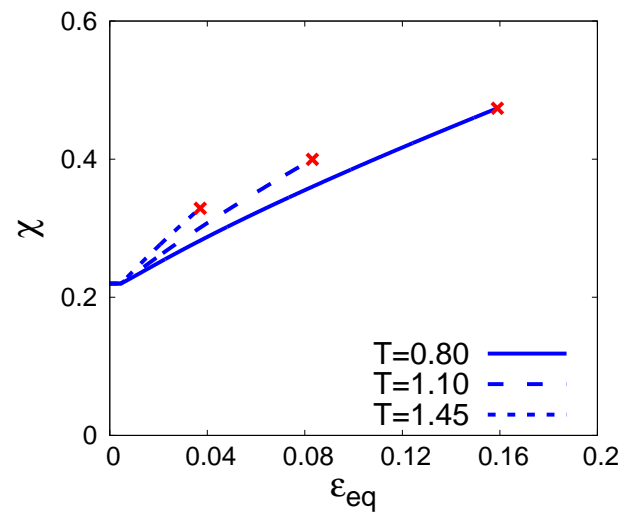

(b)

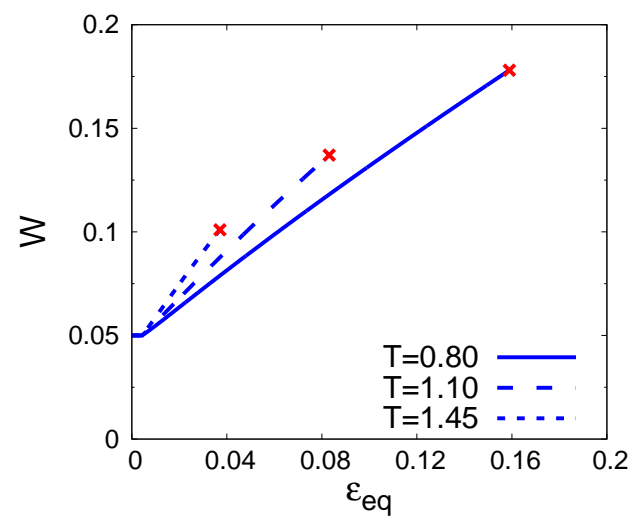

(d)

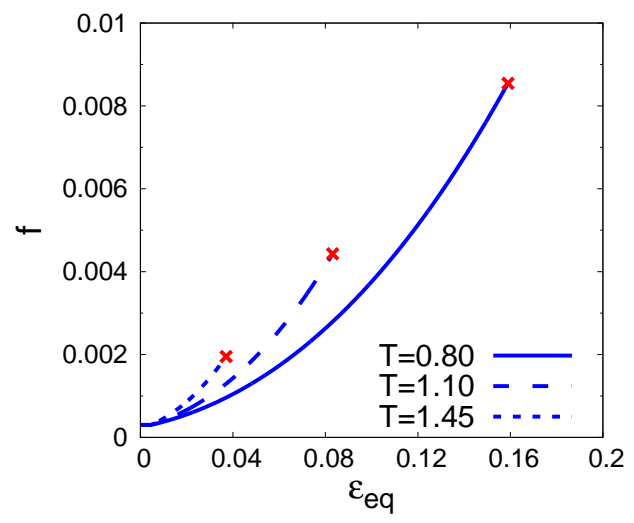

Figure 10: (a) Von Mises effective stress, $\sigma_{\text {eq }}$, versus effective strain, $\varepsilon_{\text {eq }}=\bar{\varepsilon}$, for the three triaxialities used in Fig. 9 and $f_{0}=0.0002, W_{0}=0.05$ and $\chi_{0}=0.22$. (b) Void aspect ratio, $W$, versus $\varepsilon_{\text {eq. }}$ (c) Ligament parameter, $\chi$, versus $\varepsilon_{\text {eq }}$. (b) Porosity, $f$, versus $\varepsilon_{\text {eq }}$. 
(a)

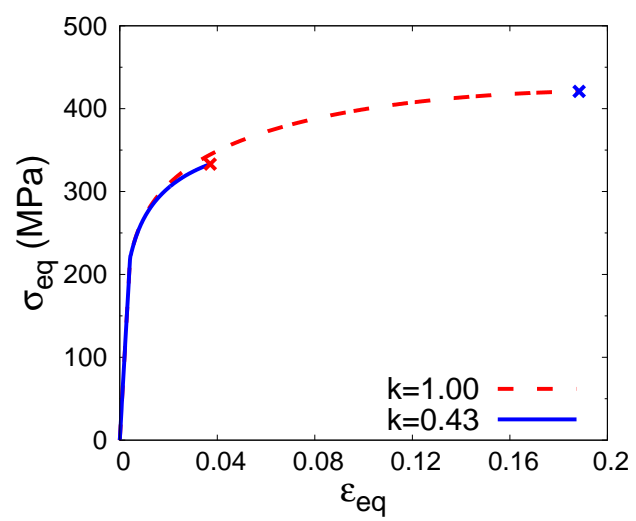

(c)

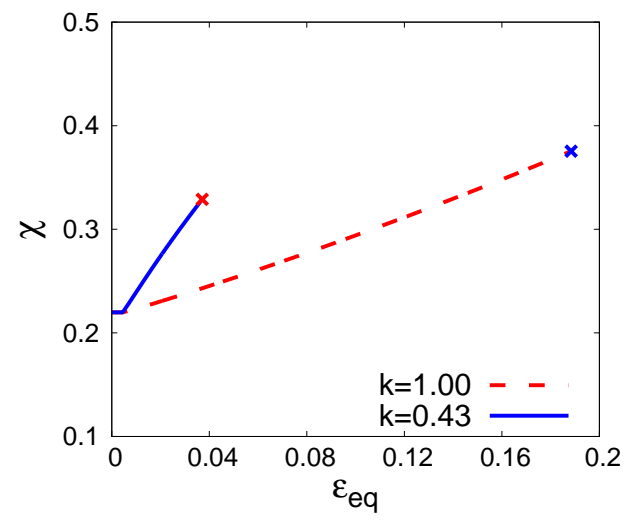

(b)

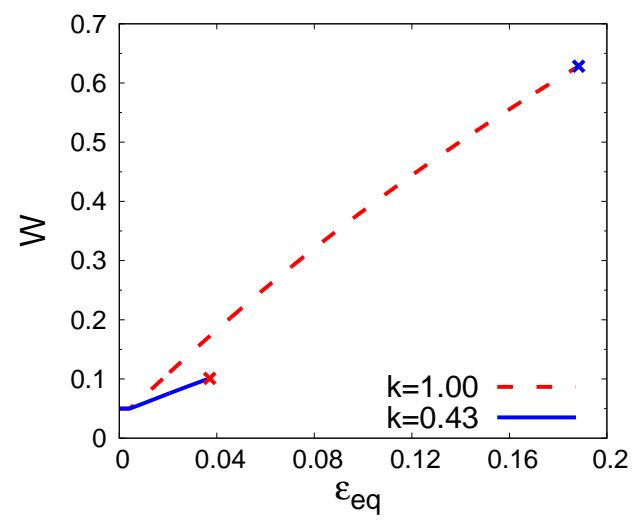

(d)

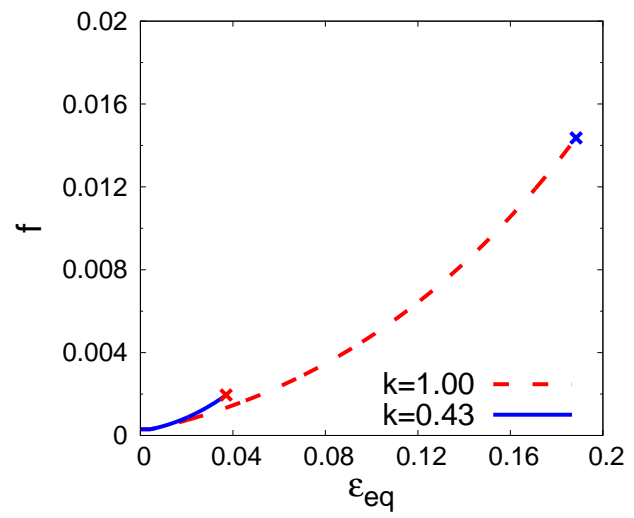

Figure 11: Effect of heuristic parameter $k$ in equation (17) on the responses shown in Fig. 10 in the special case of $T=1.45$. 
Experimental Results
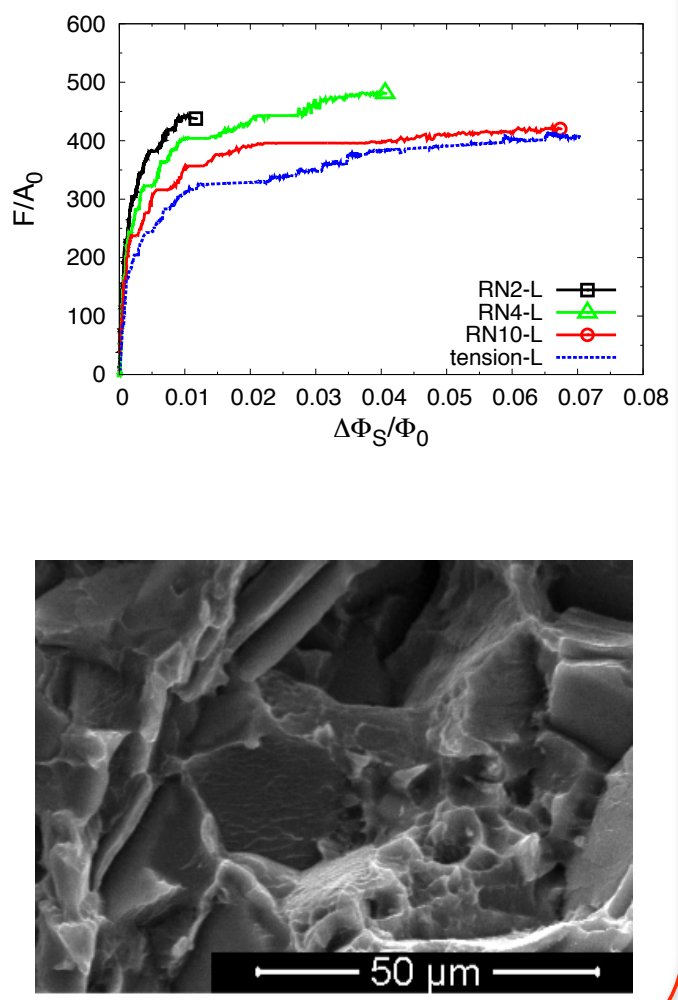

Material Parameter Identification
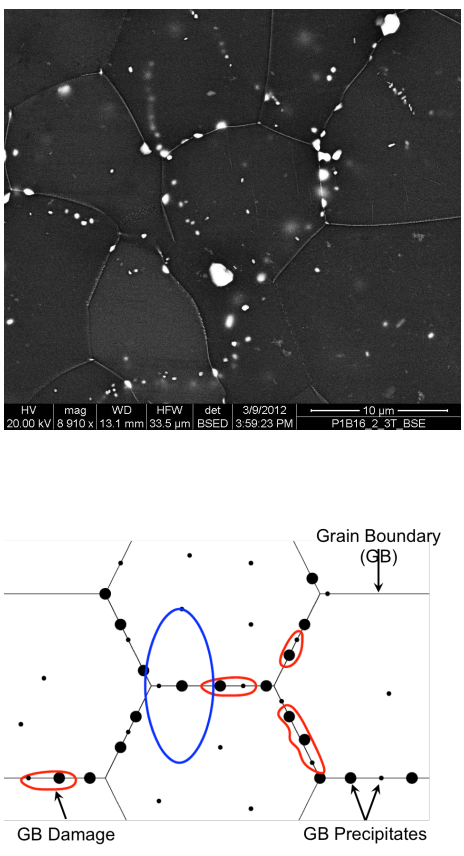

\section{Model Predictions}

Model, $\mathrm{k}=1.00$ Model, $\mathrm{k}=0.43$ Experiments $\square$

$\Delta$

$\Delta$
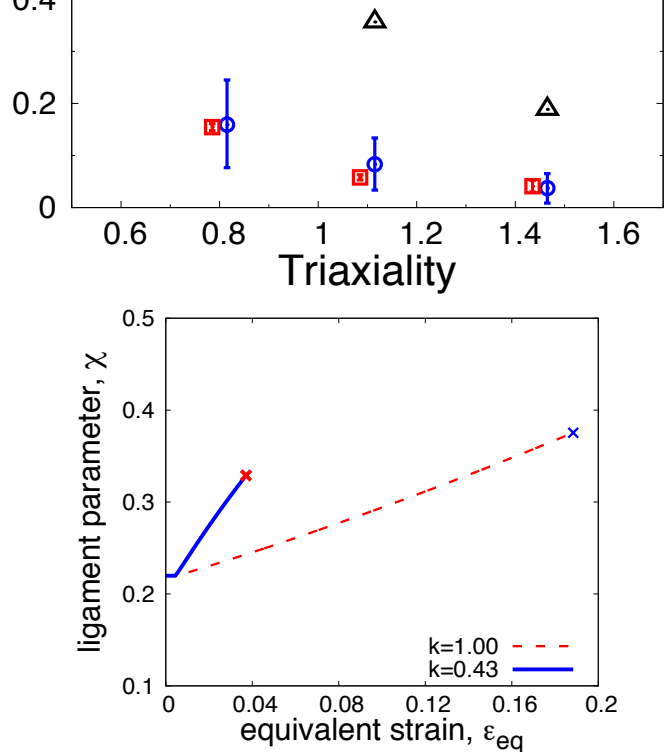